\title{
Investigations of hydrogen bonding in the poly(urethane-urea)-based mem- brane materials by using FTIR spectroscopy
}

\author{
${ }^{1}$ Aleksandra Wolińska-Grabczyk, Bożena Kaczmarczyk, Andrzej Jankowski \\ Polish Academy of Sciences, Centre of Polymer and Carbon Materials, ul. M. Curie-Skłodowskiej 34, 41-819 Zabrze, \\ Poland \\ ${ }^{1}$ Corresponding author: aleksandra.wolinska@cmpw-pan.edu.pl
}

\begin{abstract}
FTIR analysis was used to study the hydrogen bonding in 4,4'-diaminodiphenylmethane-based segmented poly(urethane-urea)s varying in the length of the poly(tetramethylene oxide) (PTMO)-based soft segments. Experiments were designed to follow the IR absorption of both the $\mathrm{NH}$ and carbonyl regions as a function of temperature in order to directly investigate the extent and strength of the hydrogen bonds, and thereby to gain some information about the possible alteration of the initial phase-segregated morphology as a result of the applied thermal treatment.
\end{abstract}

Keywords: Infrared spectroscopy, hydrogen bonding, polyurethanes.

\section{INTRODUCTION}

Thermoplastic polyurethanes are linear, segmented block copolymers that consist of alternating soft and hard segment units. It is generally accepted that the unique properties of those materials, like the strength and high elasticity, are mainly due to the ability of the segments to segregate and to form two-phase morphology ${ }^{1}$. Along with the microphase separated domains structures, polyurethanes exhibit extensive hydrogen-bonding. The proton donor is the $\mathrm{NH}$ group of the urethane linkage, whereas the hydrogen-bond acceptor may be either in the hard segment (the carbonyl of the urethane group) or in the soft segment (e. g. ether oxygen). The extent and nature of hydrogen bonding seems to be one of the most important parameters, which may affect the morphology and the final properties of polyurethanes. Therefore, it has been a subject of numerous investigations, including those performed by using infrared spectroscopy ${ }^{2-4}$. Although the validity of some previous work concerning the interpretation of the infrared changes with temperature has been questioned later on ${ }^{5}$, this method can be regarded as a very versatile technique to characterise the different hydrogen bonds and to deduce phase-separated structures in polyurethanes.

In this work FTIR thermal analysis was used to study the extent, distribution and the thermal behaviour of hydrogen bonding in the poly(tetramethylene oxide)-based poly(urethane- urea)s (PUU) used as membrane materials for gas and liquid separations. An unusual feature of these materials is that their transport properties may change depending on temperature, at which the annealing process has been performed. This is in contrast to the behaviour of the non-segmented polyurethanes and the respective polyurethanes chain extended with low molecular diols, which all show their transport characteristics to be unaltered by the similar thermal treatment ${ }^{6}$. Since we attribute this observation to some structural reorganisation taking place in the poly(urethane-urea)s during heating, the aim of this work was to investigate the temperature induced changes in the hydrogen bonding characteristics by FTIR in an attempt to discern these effects on the physical properties of PUUs.

\section{EXPERIMENTAL}

\begin{abstract}
Materials
PUUs were synthesised in N,N-dimethylformamide (DMF) solution using a two-step procedure, which involved the initial end-capping of the poly(oxytetramethylene) diol (PTMO) with 2,4-tolylene diisocyanate (2,4-TDI) followed by a chain extension with 4,4'-diaminodiphenylmethane (PP). The chemical structure of PUUs is shown in Scheme 1 and the characterisation of the obtained polymers is given in Table 1 . The details of the polyurethane synthesis have been described previously ${ }^{7}$.
\end{abstract}

Table 1. Characteristics of PUUs

\begin{tabular}{|c|c|c|c|c|}
\hline Sample code & $\begin{array}{c}\text { Composition } \\
\text { TDI/PP/PTMO }\end{array}$ & Molar mass of PTMO glycol & $\begin{array}{c}\text { Molar mass } \\
\text { of PUUs }\end{array}$ & Soft segment content [wt. \%] \\
\hline PUU-1000 & $2 / 1 / 1$ & 1000 & 50600 & 65 \\
\hline PUU-650 & $2 / 1 / 1$ & 650 & 42600 & 54 \\
\hline
\end{tabular}

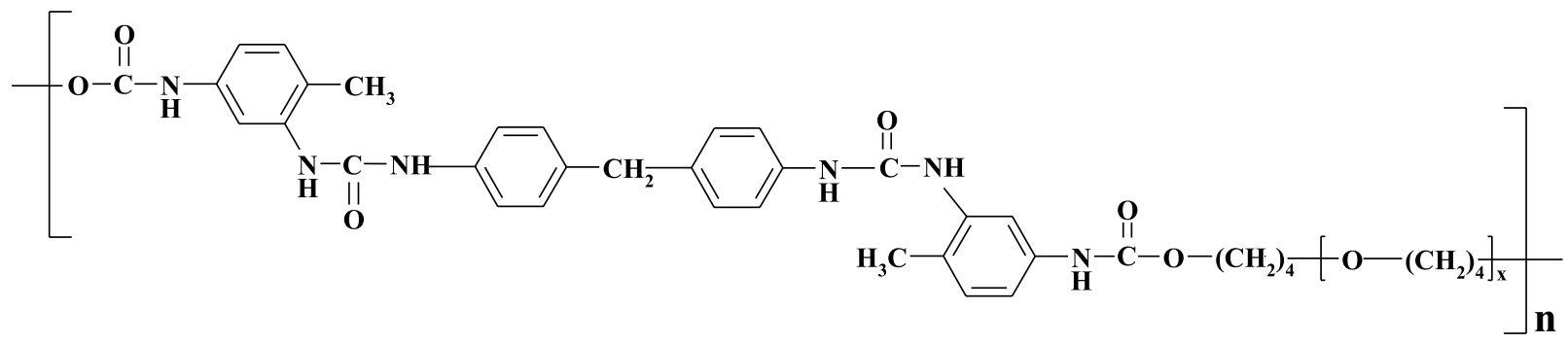




\section{Measurements}

Infrared spectra were acquired on a BIO-RAD FTS-40A Fourier transform infrared spectrometer in the range of 4000 - $700 \mathrm{~cm}^{-1}$ at a resolution of $2 \mathrm{~cm}^{-1}$ and for accumulated 40 scans. The samples investigated were prepared generally in the same way as the polyurethane-based membranes used in the transport studies. However, for the purpose of this work the 3 wt. \% solution of PUU in DMF instead of $15 \mathrm{wt}$. \% was used. The films were prepared by pouring the solution onto a potassium bromide plate and by evaporating the solvent at $60^{\circ} \mathrm{C}$ in a dry nitrogen atmosphere for 4 days. These conditions allow the polymer films with stable morphology and properties to be obtained $^{7}$. Spectra at elevated temperatures were recorded in the temperature range from 20 to $220^{\circ} \mathrm{C}$ using a Carl Zeiss Jena high-temperature control equipment. The samples were heated at the rate of $2^{\circ} \mathrm{C} / \mathrm{min}$ under nitrogen and spectra were recorded at $20 \mathrm{deg}$. intervals.

In order to compute particular peaks, a GRAMS/386 WIN IR curve fitting program was used. An interactive procedure, with a number of peaks equal eight and Gaussian-Lorentzian type of fitting curves, was chosen.

\section{Results and discussion}

The FTIR spectra of NH-stretching region and amide I region for PUU-1000 and PUU-650 are illustrated in Fig.1.

For the both investigated polyurethanes the bands ascribed to the stretching vibrations of the NH urethane and urea groups are observed in a form of a one broad band, which is centred at 3290 and $3298 \mathrm{~cm}^{-1}$ for PUU-1000 and PUU-650, respectively. This suggests that there are no free $\mathrm{NH}$ groups in the samples investigated. In the region attributed to the amide I groups, i.e. the stretching vibrations of $\mathrm{C}=\mathrm{O}$ urethane and urea groups, the broad band with several shoulders is detected for both polymers. It is well-known in literature that the infrared absorbance of H-bonded urethane or urea carbonyl appears at

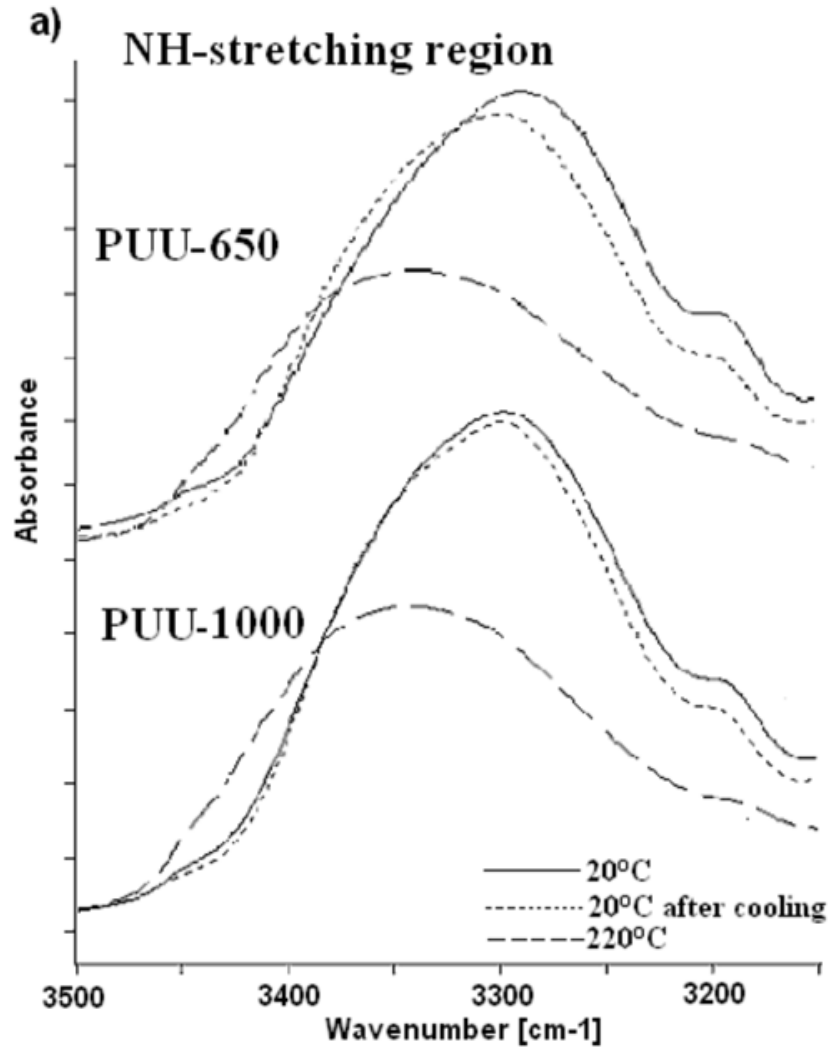

a lower wavenumber than that of free carbonyl ${ }^{8}$. Thus, in the case of PUU-1000 the two overlapping bands at 1727 and 1710 $\mathrm{cm}^{-1}$ can be assigned to the free and $\mathrm{H}$-bonded urethane $\mathrm{C}=\mathrm{O}$ groups, whereas the shoulders at about 1690 and $1667 \mathrm{~cm}^{-1}$ to the free and $\mathrm{H}$-bonded urea $\mathrm{C}=\mathrm{O}$ groups, respectively. The similar multiple overlapping bands at 1730, 1715, 1695 and $1680 \mathrm{~cm}^{-1}$ are also observed for PUU-650. On the basis of these results, it can be assumed that all $\mathrm{NH}$ groups form hydrogen bonds either with urethane and urea $\mathrm{C}=\mathrm{O}$ groups or with other groups, like the ether ones.

Comparing the spectra of PUU-1000 and PUU-650, it can be seen that in the amide I region (Fig. 1b) relative intensities of the bands corresponding to the free and $\mathrm{H}$-bonded urethane $\mathrm{C}=\mathrm{O}$ groups are different for both polymers. The main component of the PUU-650 carbonyl band is the H-bonded urethane, whereas for PUU-1000 the components assigned to the free and $\mathrm{H}$-bonded urethane $\mathrm{C}=\mathrm{O}$ groups are comparable. On the other hand, the presence of the distinct bands corresponding to the free $\mathrm{C}=\mathrm{O}$ group in the IR spectra of the both PUUs investigated indicates that both polymers are not fully microphase separated and that there are some hard segments mixed into the soft phase. This is in accordance with the generally observed tendency ${ }^{1}$ and with the results of our previous investigations ${ }^{7}$ that the complete phase separation occurs for polyurethanes with longer segments.

To investigate the changes in the hydrogen bonds distribution resulting from the thermal treatment, the spectra of PUUs were recorded as a function of increasing and the subsequent decreasing temperature, and analysed with respect to the peak shape, intensity, and the position shift. In Fig.1. the representative spectra measured at $20^{\circ} \mathrm{C}, 220^{\circ} \mathrm{C}$, and after cooling to $20^{\circ} \mathrm{C}$ are shown. As it can be seen from Fig.1a, maximum of the band corresponding to the stretching vibrations of $\mathrm{NH}$ groups shifted with temperature to higher wavenumbers due to the elongation of the hydrogen bonds. However, hydrogen bonds can still be detected even at $220^{\circ} \mathrm{C}$. The similar peak shift occurs in the

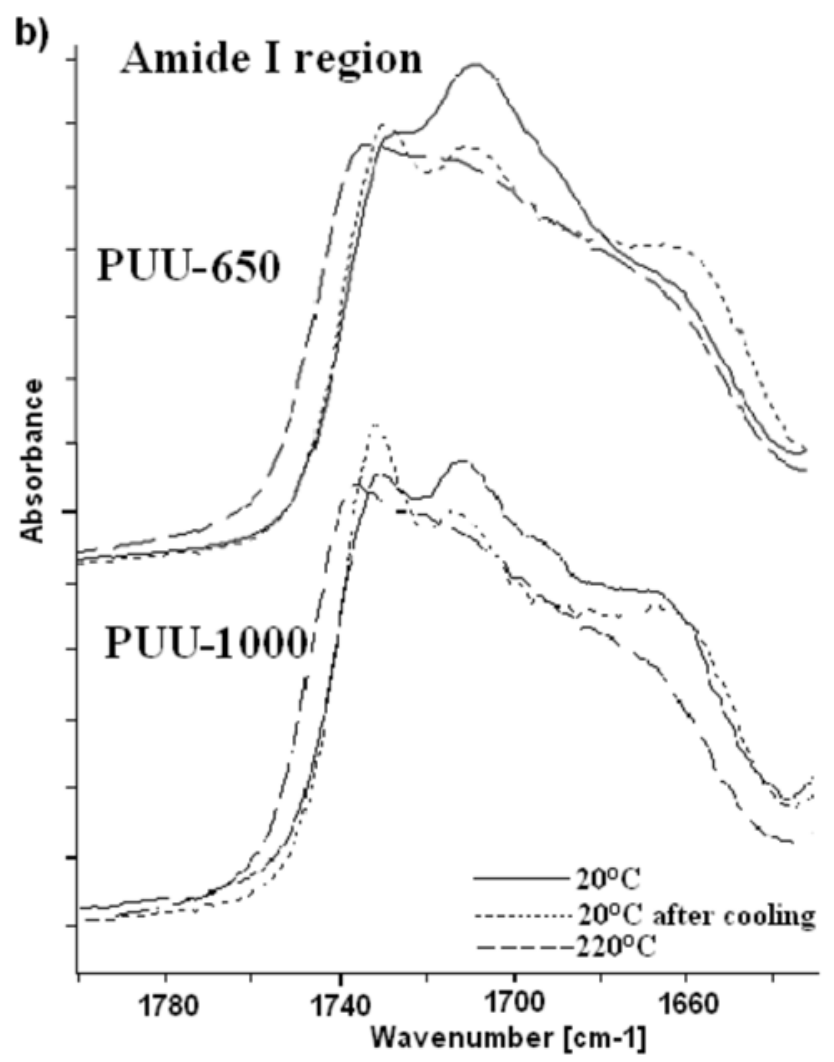

Figure 1. FTIR spectra of PUUs recorded at $20^{\circ} \mathrm{C}, 220^{\circ} \mathrm{C}$ and after cooling to $20^{\circ} \mathrm{C}$ 
Table 2. Deconvolution results in the amide I region

\begin{tabular}{|c|c|c|c|c|c|c|c|c|}
\hline \multirow{2}{*}{ Sample } & \multicolumn{3}{|c|}{$\mathrm{C}=\mathrm{O}$ urethane groups $\left[\mathrm{cm}^{-1}\right]$} & \multicolumn{4}{c|}{$\mathrm{C}=\mathrm{O}$ urea groups $\left[\mathrm{cm}^{-1}\right]$} \\
\cline { 2 - 10 } & Free & \multicolumn{3}{|c|}{ H-Bonded } & Free & \multicolumn{3}{c|}{ H-Bonded } \\
\cline { 2 - 10 } & & disordered A & disordered B & ordered & & disordered A & disordered B & ordered \\
\hline PUU-1000 & 1738 & 1732 & 1723 & 1713 & 1699 & 1683 & 1670 & 1658 \\
\hline PUU-650 & 1737 & 1731 & 1720 & 1713 & 1699 & 1690 & 1677 \\
\hline
\end{tabular}

amide I region. However, the spectra in Fig.1b indicate substantial changes in the shape of the carbonyl band at higher temperatures for both polymers. It should be noted that the spectra are plotted on an absolute absorbance scale, and consequently, the changes in wavenumber and relative area can be directly compared. The spectrum of PUU- 650 at $20^{\circ} \mathrm{C}$ is characterised by a strong absorbance at $1710 \mathrm{~cm}^{-1}$. When the sample is heated to $220^{\circ} \mathrm{C}$, this band, being assigned to the $\mathrm{H}$-bonded urethane carbonyl, decreases in absolute intensity, whereas that at 1727 $\mathrm{cm}^{-1}$ assigned to the free urethane carbonyl exhibits relatively smaller changes. The similar behaviour is observed for the PUU-1000 sample. After cooling, the carbonyl regions of both polymers have been found to differ significantly, showing the increase in absolute intensity of the free urethane carbonyl band. It can suggest that some of the hydrogen-bonded carbonyls transformed into free carbonyls as a result of the thermal treatment.

Considering the fact that bands in that region are strongly overlapped, the curve fitting calculation was used to monitor in details the changes of the particular band area and position during heating and cooling scans. The obtained results of the curve fitting concerning the position of the bands for the both initial samples at $20^{\circ} \mathrm{C}$ are given in Table 2. Curve fitting was limited to the spectral data of the amide I region between 1780
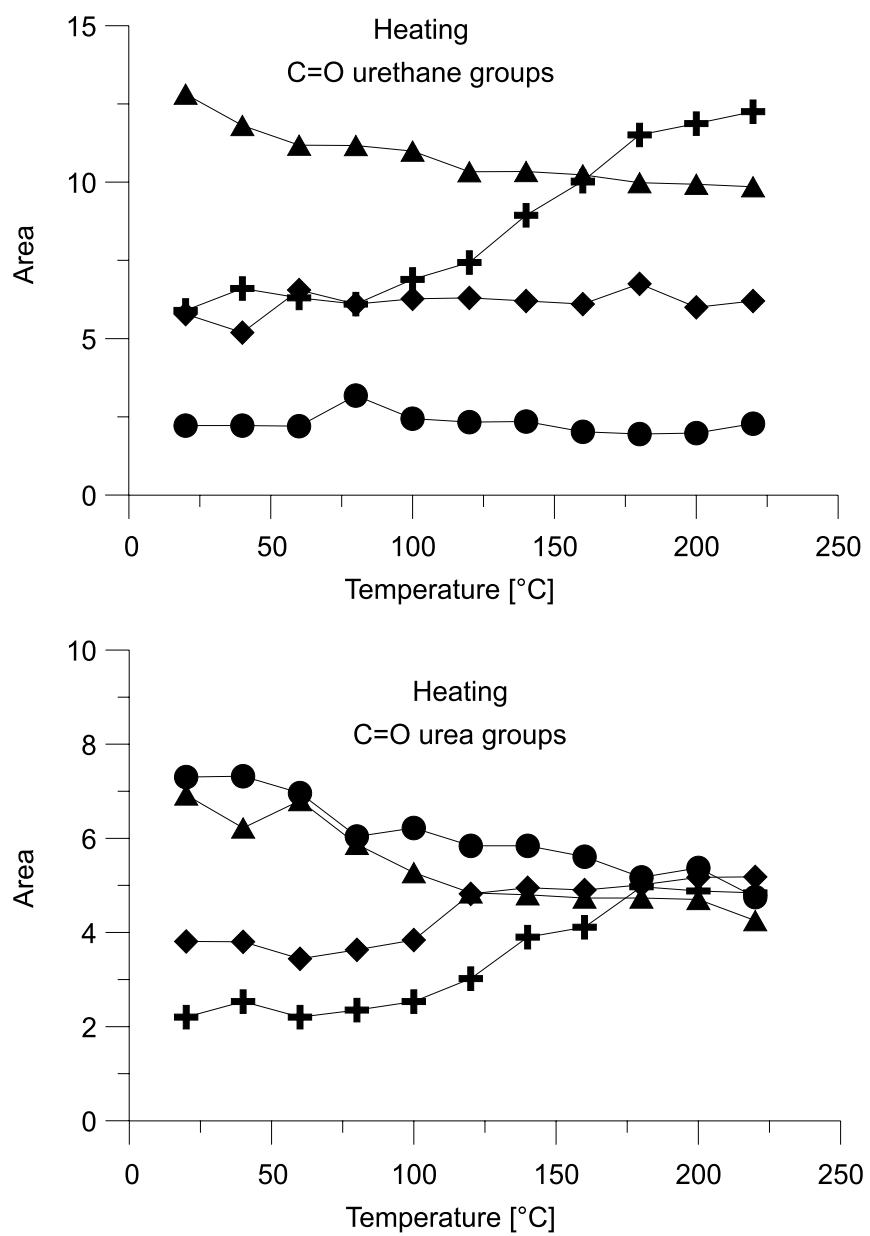

and $1640 \mathrm{~cm}^{-1}$. It was found that good fitting results could be obtained with the assumption that the number of peaks equals eight. The obtained results can be interpreted as associated with urethane and urea carbonyls not involved in hydrogen bonding, and with those involved in hydrogen bonds of differing geometries. As a first approximation, the bands have been assigned to free carbonyls, disordered $\mathrm{H}$-bonds of $\mathrm{A}$ and $\mathrm{B}$ type in different environments, and the ordered hydrogen bonds as named in an order of decreasing wavenumbers. Similar deconvolution results concerning polyamides and polyurethanes have also been reported in literature $\mathbf{2 , 8 , 9}$.

The curve fitting data concerning the changes in the areas of the particular bands as a function of temperature are plotted in Fig. 2 and 3.

In the data presented in those figures, a progressive increase in the areas of the bands corresponding to the free urethane and free urea carbonyls with increasing temperature is observed for both PUUs. Upon samples cooling, the area of the respective bands decreases, however, in the case of PUU-1000 the bands intensities do not reach the level determined for the initial samples. On the other hand, the opposite behaviour is displayed by H-bonded carbonyls, both ordered and disordered, the amount of which decreases during heating and only slightly increases upon cooling. These observations must be associated with the
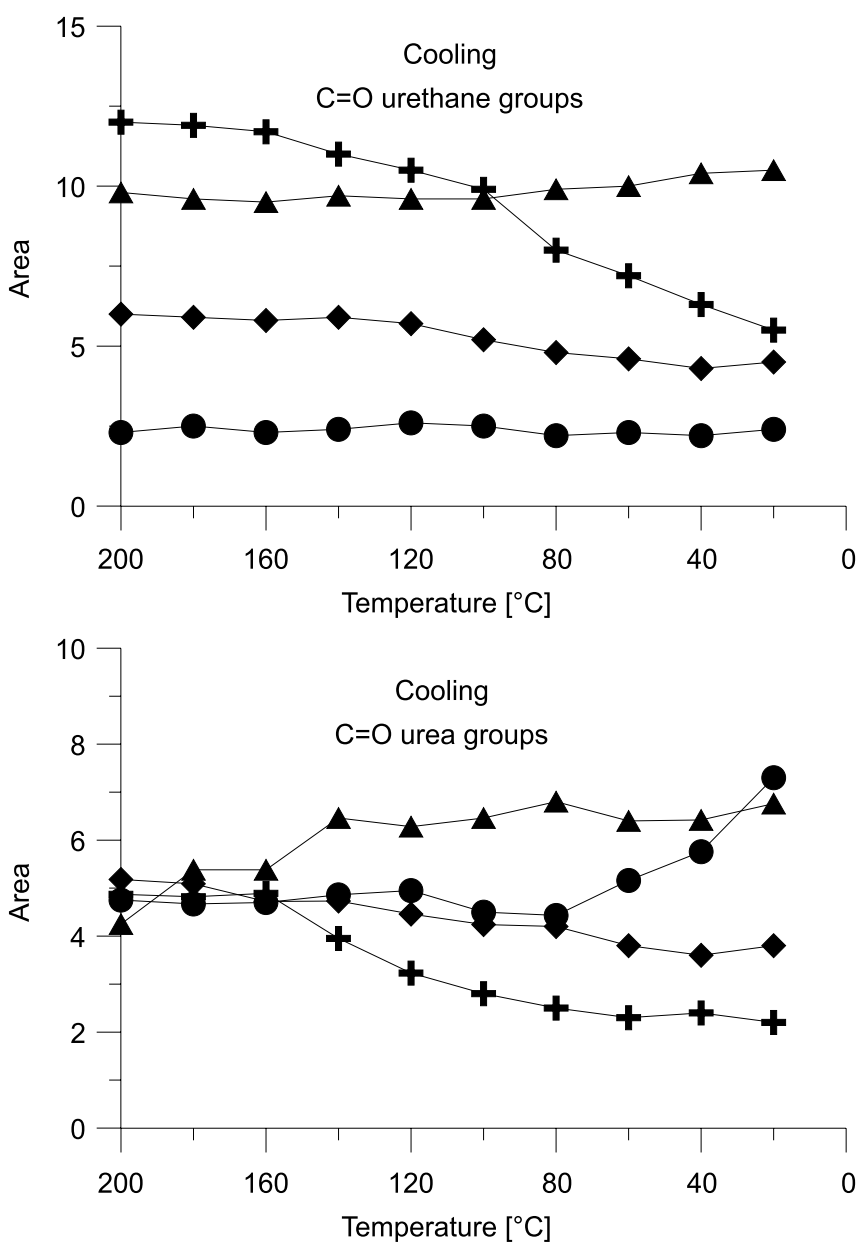

Figure 2. Changes in the areas of bands in amide I region during heating and cooling for PUU-650: +- free, $\bullet-\mathrm{H}-\mathrm{bonded}$ disordered $\mathrm{A}, \bullet-\mathrm{H}$-bonded disordered $\mathrm{B}, \mathbf{\Delta}-\mathrm{H}$-bonded ordered 

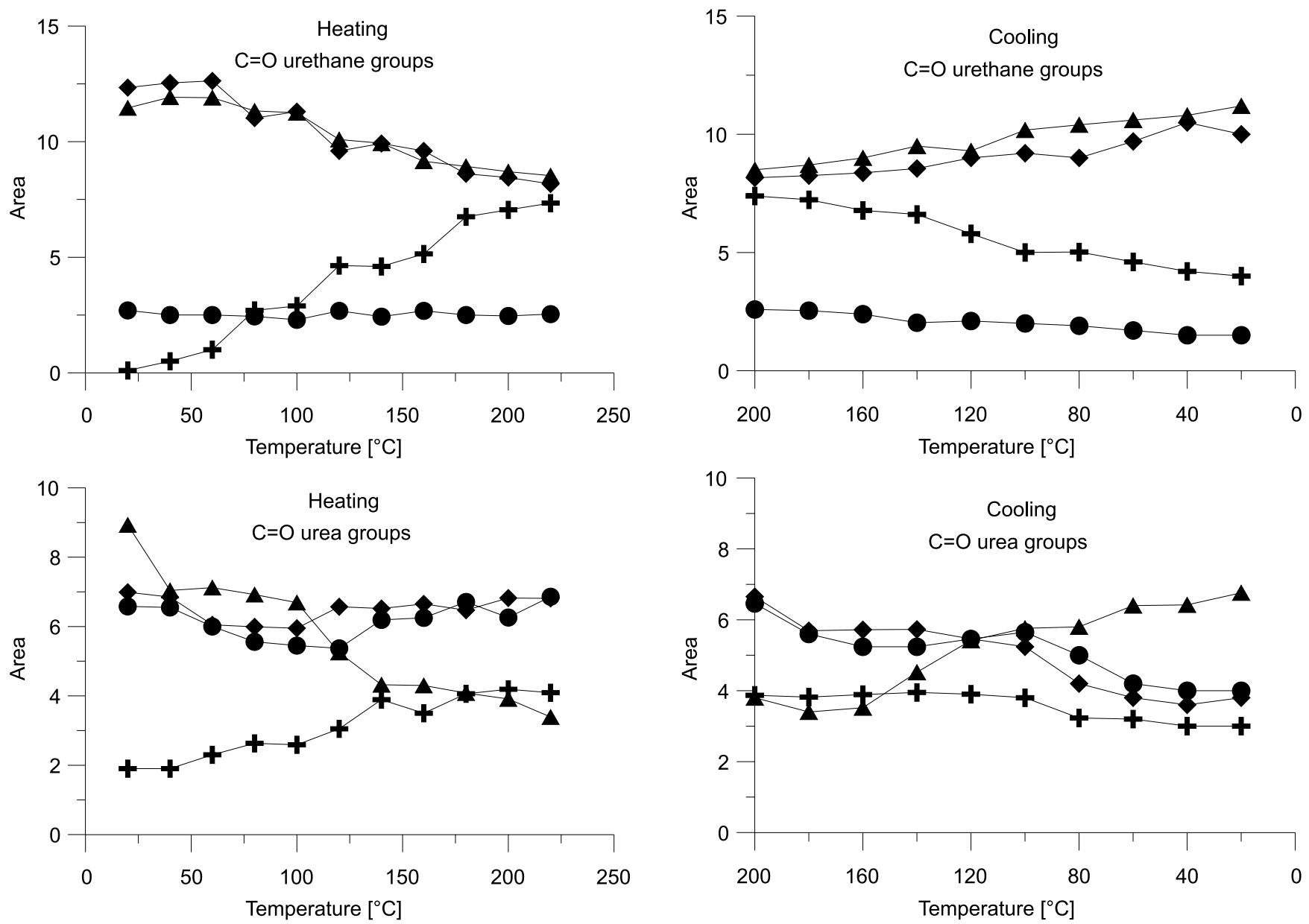

Figure 3. Changes in the areas of the bands in amide I region during heating and cooling for PUU-1000: + - free, - - H-bonded disordered A, - - H-bonded disordered B, $\wedge$ - H-bonded ordered

disruption of hydrogen bonding in some segregated hard domains and the hard segments pulling out from the hard domains into the soft matrix. As a consequence, the structure transformation is expected to take place, which persists after the thermal treatment, and which may be responsible for the altered transport properties of those polymers ${ }^{6}$.

\section{ACKNOWLEDGEMENT.}

This work was financed by the grant No N 205070 31/3205 given by the Ministry of Science and Higher Education of Poland from its means for scientific research for the years 20062008.

\section{LITERATURE CITED}

1. Velankar, S. \& Cooper, S.L. (1998). Microphase Separation and Rheological Properties of Polyurethane Melts. 1. Effect of Block Length. Macromolecules 31, 9181 - 9192. DOI: $10.1021 / \mathrm{ma} 9811472$.

2. Coleman, M.M., Lee, K.H., Skovranek, D.J. \& Painter, P.C. (1986). Hydrogen bonding in polymers. 4. Infrared temperature studies of a simple polyurethane, Macromolecules, 19, 2149 - 2157. DOI: 10.1021/ma00162a008.

3. Lee, H.S., Wang, Y.K. \& Hsu, S.L. (1987). Spectroscopic analysis of phase separation behavior of model polyurethanes. Macromolecules, 20, 2089 - 2095. DOI: 10.1021/ma00175a008.

4. Lee, H.S. \& Hsu, S.L. (1989). An analysis of phase separation kinetics of model polyurethanes. Macromolecules, 22, 1100 - 1105. DOI: 10.1021/ma00193a017.

5. Coleman, M.M., Skovranek, D.J., Howe, S.E. \& Painter, P.C. (1985). On the validity of a commonly employed infra- red procedure used to determine thermodynamic parameters associated with hydrogen bonding in polymers, Macromolecules, 18, 299 - 301. DOI: 10.1021/ma00144a033.

6. Wolińska-Grabczyk, A., Wrzalik, R.\& Jankowski, A., in preparation.

7. Wolińska-Grabczyk, A. (2004). Relationships between permeation properties of the polyurethane-based pervaporation membranes and their structure studied by a spin probe method. Polymer 45. 4391 - 4402. DOI: 10.1016/ j.polymer.2004.04.039.

8. Skovranek, D.J., Painter, P.C. \& Coleman, M.M. (1986). Hydrogen bonding in polymers. 2. Infrared temperature studies of nylon 11. Macromolecules. 19, 699 - 705. DOI: 10.1021/ma00157a037.

[9] Teo, L.S., Chen, Ch.Y. \& Kuo, J.F. (1997). Fourier Transform Infrared Spectroscopy Study on Effects of Temperature on Hydrogen Bonding in Amine-Containing Polyurethanes and Poly(urethane-urea)s'. Macromolecules, 30, 1793 - 1799. DOI: 10.1021/ma961035f. 